\title{
The formation mechanism of EVAL membranes prepared with or without the nonsolvent absorption process
}

\author{
Tai-Horng Young*, Yao-Huei Huang, Yen-Shih Huang \\ Institute of Biomedical Engineering, College of Medicine and College of Engineering, National Taiwan University, Taipei 10016, Taiwan
}

Received 30 July 1999; received in revised form 11 November 1999; accepted 13 December 1999

\begin{abstract}
In this work, the effect of the nonsolvent absorption from the atmosphere into the casting solution on the membrane structure in the poly (ethylene-co-vinyl alcohol) (EVAL)-DMSO-water system was studied via scanning electron microscopy. If the casting solution was directly immersed into a water bath, the membrane showed a typically asymmetric structure dominated by liquid-liquid demixing process. As the water vapor as a coagulant, the membrane showed another asymmetric structure: porous surface with cellular pores supported by a homogeneous particles-bonded sublayer. The particle structure is representative of crystallization-induced solid-liquid demixing from a homogeneous mixture. If the water absorption time was decreased, a progressive change in the membrane morphologies containing features from both liquid-liquid demixing and solid-liquid demixing phase separations could be observed. This suggests that different types of membranes controlled by different phase separations should have different composition paths in the phase diagram even though they had the same phase diagram. Therefore, this difference in membrane structure prepared with or without nonsolvent absorption was discussed by taking into account the effect of the diffusion kinetics of the membrane formation system. (C 2000 Elsevier Science B.V. All rights reserved.
\end{abstract}

Keywords: Membrane morphology; Absorption; Crystallization; Liquid-liquid demixing

\section{Introduction}

Nowadays polymeric membranes are produced mainly by the nonsolvent-induced phase inversion process [1]. In this process, a casting solution consisting of polymer and solvent is brought into contact with nonsolvent. By exchange of solvent and nonsolvent causes the polymer solution to phase-separate, which forms the membrane. With crystallizable polymers, two types of phase separation may occur during the membrane formation. Liquid-liquid demixing results

\footnotetext{
${ }^{*}$ Corresponding author. Tel.: $+886-2-2397-0800$ extn. 1455; fax: +886-2-2394-0049.

E-mail address: thyoung@ @a.mc.ntu.edu.tw (T.-H. Young)
}

in the typical cellular morphology with pores from polymer-poor phase surrounded by the membrane matrix from polymer-rich phase [1]. Solid-liquid demixing is from crystallizable segments of the polymer to form membranes by linking of particles [2-7]. For example, Wijmans et al. prepared membranes with linking of particles from a casting solution of poly (2,6-dimethyl-1,4-phenyleneoxide) in mixtures of trichloroethylene, 1-octanol, and methanol [2]. Bulte et al. [3] and Cheng et al. [4,5] prepared polyamide membranes with a spherulitic structure by solid-liquid demixing in the immersion precipitation process. Witte et al. did the same for poly-L-lactide with dioxane or $N$-methyl pyrrolidone as solvents and with methanol or water as nonsolvents [6]. Recently, Cheng 
and coworkers manufactured particulate microporous membranes by isothermal immersion precipitation from 1-octanol-dimethylformamide-poly(vinylidene fluoride) system [7]. Therefore, for membranes fabricated from precipitation of crystallizable polymers, crystallization processes, other than liquid-liquid demixing, can also induce the membrane formation and are important for the membrane structure.

Poly (ethylene-co-vinyl alcohol) (EVAL) is a semicrystalline copolymer. In our laboratory, we have prepared EVAL membranes characterized by a packed bed of nearly equal-diameter particles in several ways [8-14]. Such particulate membranes have proved useful in microfiltration [8], plasma protein separation [9], and cell culture [10]. The basic principle of controlling membrane structures from a crystallizable polymer is to manage the competition between liquid-liquid demixing process and solid-liquid demixing process. Since crystallization is a slow process in comparison with liquid-liquid demixing, the method to prepare the crystallization-controlled membrane structure is the addition of a substantial amount of solvent to the coagulation bath to reduce the rate of solvent-nonsolvent exchange [11]. In this case, liquid-liquid demixing is not possible until a certain period of time has elapsed. Therefore, the time available for the membrane solution within the solid-liquid demixing region is large enough to initiate solid-liquid demixing. Alternatively, precipitation of a casting solution is brought about in 1-octanol [9]. Ordinarily, by carrying out the precipitation in 1-octanol or other alcohols [12,13], liquid-liquid demixing can be effectively suppressed and the binodal boundary is entered sluggishly. In the meantime crystallization, rather than liquid-liquid demixing, starts to dominate the membrane structure. The third technique is to prepare membranes by evaporating the casting solution for a proper period [14]. The phase separation proceeds slowly via solid-liquid demixing and thus leads to a particulate morphology in the membrane. Based on these methods, the particulate morphology obtained in EVAL membrane is mainly attributed to the diffusion kinetics in a delay time for the membrane solution to give solid-liquid demixing.

This paper reports another technique to prepare particulate membranes from EVAL polymer. Phase separation of the casting solution was brought about by imbibing nonsolvent from the vapor phase. We found the membrane structure combined with thin porous surface and thick particulate support. Although there are several reports describing the effect of nonsolvent vapor on the membrane structure [15-17], their research efforts are dedicated to amorphous polymers. These concepts are, however, inadequate for interpreting the complex morphology of membranes made from crystallizable polymers since their phase separation can be induced by the processes of liquid-liquid demixing only. Thus, the absorption of nonsolvent vapor has been used for the first time to yield membranes exhibiting characters from both types of phase separations.

To clearly understand the formation mechanism of EVAL membranes, the membrane structure prepared with an absorption process was compared to that prepared by a direct immersion without an absorption process. The equilibrium phase behavior of this membrane formation system has been described previously (Fig. 1) [18]. Since they have the same phase diagram, the membrane structures dictated by either type of phase separation process are made possible by considerations of the kinetic (mass transfer) aspects of the immersion-precipitation process. Therefore, an attempt was made to correlate the membrane morphology with the diffusion kinetics of the membrane formation system. Basically, this work is a rather fundamental study to gain the information on the pore and particle formation in the membranes from crystallizable polymers in general.

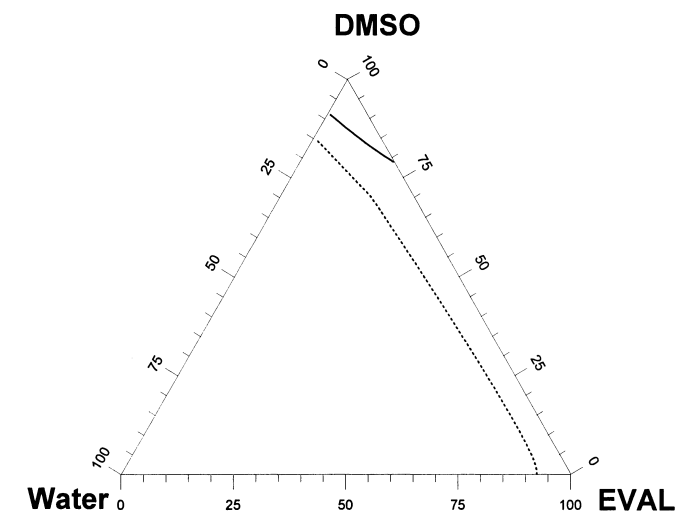

Fig. 1. Phase diagram of water-DMSO-EVAL at $25^{\circ} \mathrm{C}$ [18]. (—: equilibrium crystallization line; ----: binodal boundary.) 


\section{Experiments}

\subsection{Membrane preparation}

Membranes were prepared by using EVAL (105A, Kuraray Co. Ltd., Japan) having an average ethylene content of 44 mole\%. DMSO of extra pure reagent grade (Nacalai Tesque, Kyoto, Japan) was used as received. Water was double distilled and de-ionized before used.

An appropriate amount of EVAL was dissolved in DMSO to form a $15 \mathrm{wt} . \%$ solution. This solution was dispersed uniformly on a glass plate (ca. $100 \mu \mathrm{m})$ by an autocoater (KCC303, RK Print-Coat Instruments, UK). Membranes were prepared by directly immersing the casting solution into a water bath or subjected to a two-stage process of precipitation. For the two-stage process of precipitation, the casting solution was placed in a hood at room temperature to absorb water molecules from the atmosphere under the relative humidity of $75 \%$, then, immersed into a water bath to remove DMSO to fulfill the overall membrane. In order to investigate the effect of the water absorption time on the membrane structure, the water absorption process was carried out at three different times, 1, 3 and 5 min.

\subsection{Membrane characterization}

The morphologies of different faces of the membranes were examined using a scanning electron microscope (SEM) (S-800, Hitachi, Japan). The membranes were freeze dried, then frozen in liquid nitrogen and fractured to expose the cross-sectional areas. The dried sample were gold coated and viewed with SEM at $20 \mathrm{kV}$.

\section{Results and discussion}

\subsection{Membrane morphology}

In Fig. 2, morphologies of the membrane are shown for direct immersion of casting solution into a coagulation bath, where DMSO was employed as the casting solvent and water was used as the gelation medium. A typical asymmetrical structure consisting of a dense skin layer (Fig. 2(a)) and a sublayer with finger-like macrovoids (Fig. 2(b)) was observed. At higher magnification, it was revealed cellular morphologies enclosed in polymer matrix characteristic of amorphous membranes (Fig. 2(c)). Therefore, the phase separation process dominating this membrane formation is liquid-liquid demixing.
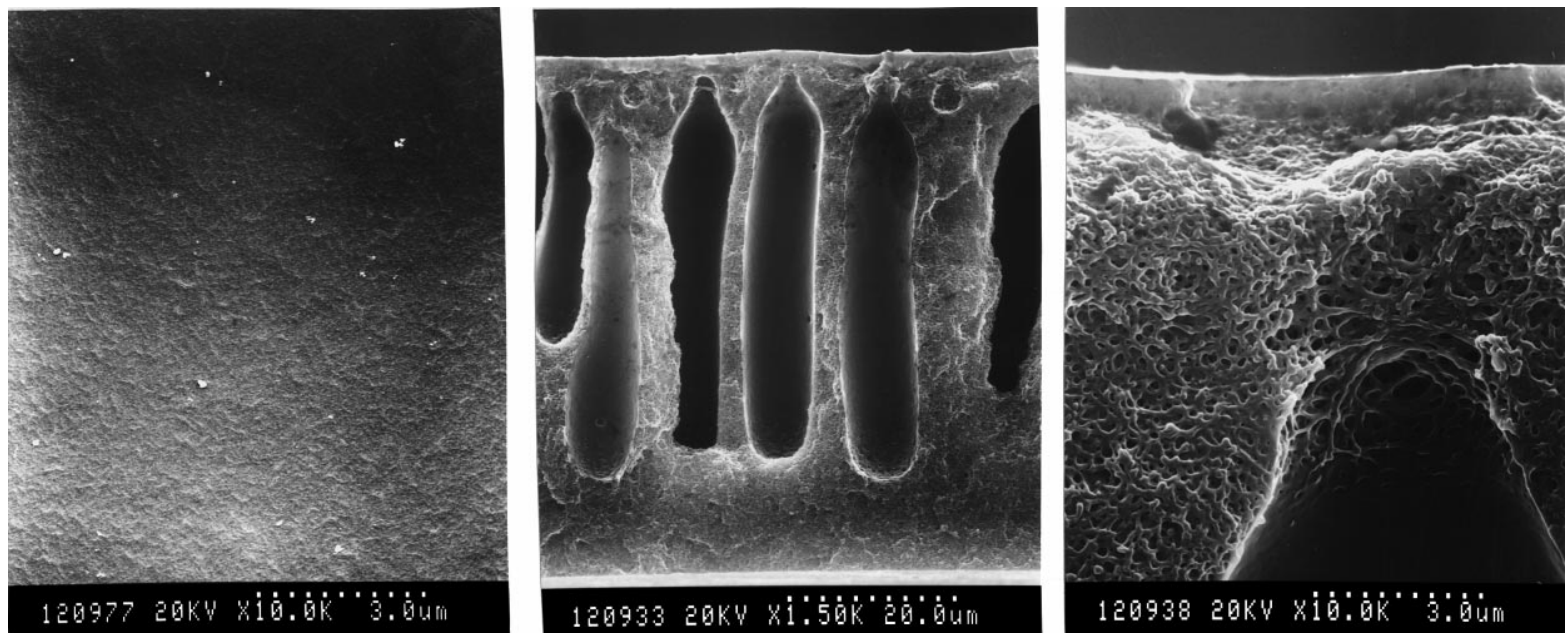

Fig. 2. SEM photographs of the EVAL membrane directly immersed in a water bath. (a) top surface; (b) cross-section; (c) magnification of (b) near the top layer. 
However, the binodal boundary is below the equilibrium crystallization line from the phase diagram of water-DMSO-EVAL at $25^{\circ} \mathrm{C}$ as shown in Fig. 1 . One should expect crystallization-induced morphology in the membrane because the membrane solution is in a metastable state initially with respect to crystallization, and then with respect to liquid-liquid demixing during membrane formation process. But typical morphology characteristic of polymer crystallization (i.e., particulate, discussed previously [2-7]) was not observed within this membrane. Hence, the membrane structure is contrary to the prediction on basis of the thermodynamic point of view.

The effect of the absorption of water molecules into the casting solution on the membrane structure under various absorption times is presented in Figs. 3-5. When $5 \mathrm{~min}$ of absorption time was employed, a complete change in morphology could be observed, as shown in Fig. 3. The membrane had a cellular surface (Fig. 3(a)) supported by a porous sublayer without any macrovoid existing (Fig. 3(b)). The cellular morphology in the membrane surface suggests that liquid-liquid demixing occurred at the beginning of the absorption-inducing phase separation process. Therefore, fast precipitation is expected at the membrane surface. On the other hand, the membrane (at higher magnification) showed uniform particles distributed in the sublayer (Fig. 3(d)) and located close to the membrane surface (Fig. 3(c)). Although the exact nature of particles has not yet been satisfactorily resolved, the particle structure is representative of crystallization-induced solid-liquid demixing from a homogeneous mixture and similar behavior was observed with other membrane formation systems [2-7].

Subsequently, membranes with absorption times of 1 and $3 \mathrm{~min}$, respectively, were prepared to observe what happens at shorter absorption times. When 1 min of absorption time was employed, the membrane still showed an asymmetrical structure (Fig. 4). However, this membrane formed superficial pores on the top surface (Fig. 4(a)). This suggests that absorbed water molecules rapidly initiated liquid-liquid phase separation. On the other hand, the cross-section of this membrane represented the completely cellular structure, but finger-like macrovoids were no longer present. (Fig. 4(b)). Therefore, a very short absorption time suggests a favorable condition for sponge-like membrane formation but unfavorable for finger-like membrane formation. Fig. 5 presents the top and cross-sectional views of the membrane prepared with absorption time of $3 \mathrm{~min}$. The top surface was like to be broken up by the growing pores and these pores penetrated to the sublayer (Fig. 5(a)). The cross-section of this membrane represents both cellular pores and particles were formed as shown in Fig. 5 (b). The magnified cross-sectional view shown in Fig. 5(c) indicates particles seemed to emerge from distorted pore wall. This implies that the membrane formation is not dictated by liquid-liquid demixing only. Particle aggregate formation due to crystallization has become a competitive or even predominant phase separation mechanism. Therefore, liquid-liquid demixing and crystallization occurred nearly simultaneously. In such a case, the formed morphology exhibits features from both types of phase transformations. Overall, membranes prepared with shorter water absorption times represented the intermediate structures between membranes prepared without and with $5 \mathrm{~min}$ of water absorption process.

\subsection{Membrane formation mechanism}

In fact, the phase diagram is only a thermodynamic description of the system in equilibrium determining the overall phase distribution. Due to the long configuration relaxation time of polymer chains in viscous solutions, the kinetic parameters become important in determining the membrane structure. The diffusion problem of the ternary system with a moving boundary during membrane formation process is very complex. Several investigators have described the diffusion of solvent and nonsolvent in membrane solutions by a simplified mass transport model [5,11,19-22]. According to the model of Reuvers et al. [21], instantaneous and delayed demixing processes can be distinguished. If the composition profile in the membrane solution immediately crosses the binodal of the nonsolvent-solvent-polymer system after immersion of the solution into a nonsolvent bath, liquid-liquid demixing takes place immediately. On the other hand, if it takes a certain time for the composition profile to cross the binodal, the onset of liquid-liquid demixing is delayed. Unfortunately, a mass transport model only predicts the time at which a concentration path first crosses the binodal curve 
(a)

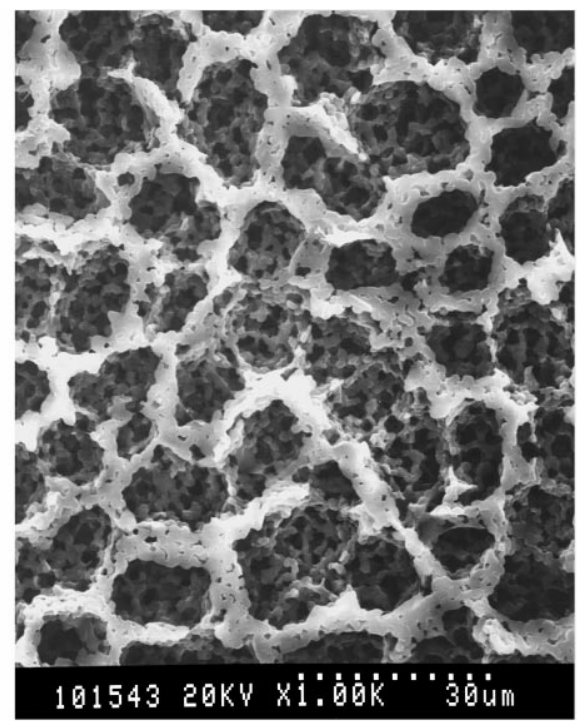

(c)

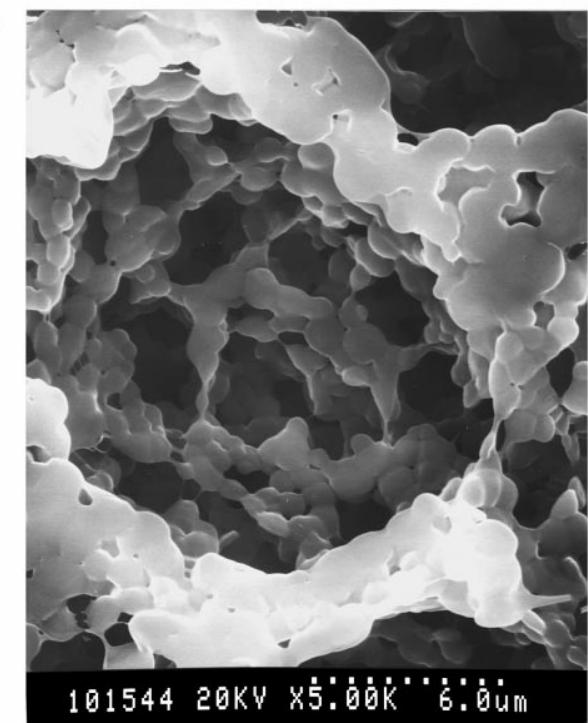

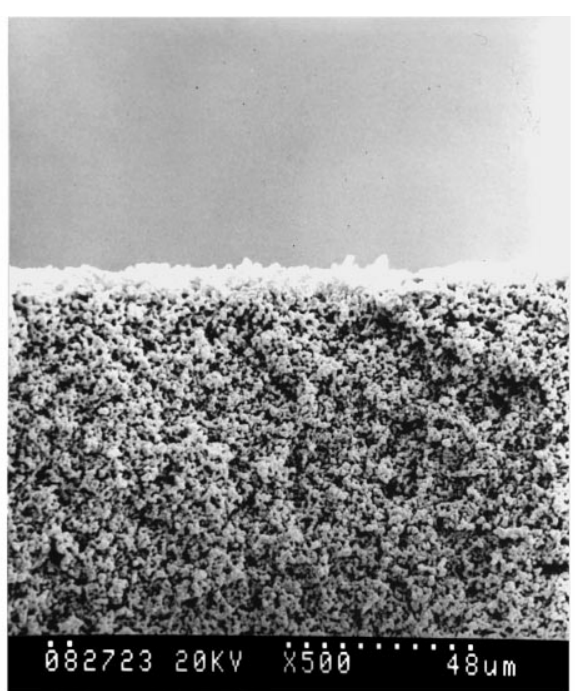

(b)

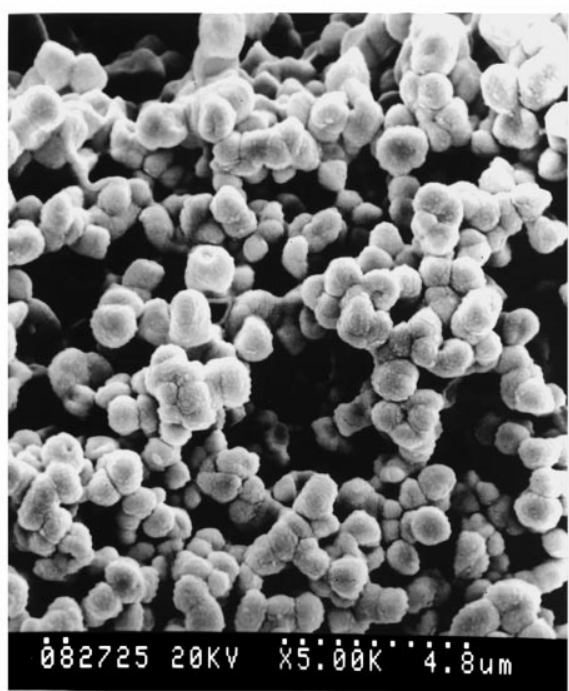

(d)

Fig. 3. SEM photographs of the EVAL membrane prepared with 5 min of absorption process at $21^{\circ} \mathrm{C}$ under the relative humidity of $75 \%$ followed by immersion into a water bath (a) top surface; (b) cross-section; (c) magnification of (a); (d) magnification of (b).

in the computation and all compositions existing in the polymer solution at this time. The prediction of the composition changes and its relation to the ultimate membrane structure after this time is beyond the scope of a mass transport model.

For an asymmetric membrane, it is reasonable to analyze the membrane formation mechanism based on a two-step process [23], i.e., the toplayer and the sub- layer have different diffusion kinetics to lead to different morphologies. With the casting solution immersed into the coagulation bath, the toplayer is formed first at the casting solution-nonsolvent interface. Due to the strong interaction between DMSO and water, the moment the casting solution and water come into contact, DMSO in the casting solution desolvates rapidly into the coagulation bath. However, only relatively 
(a)

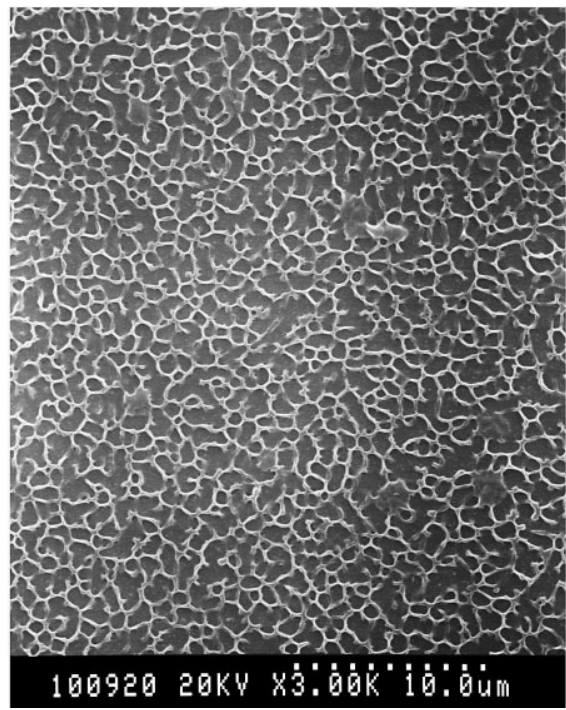

(b)

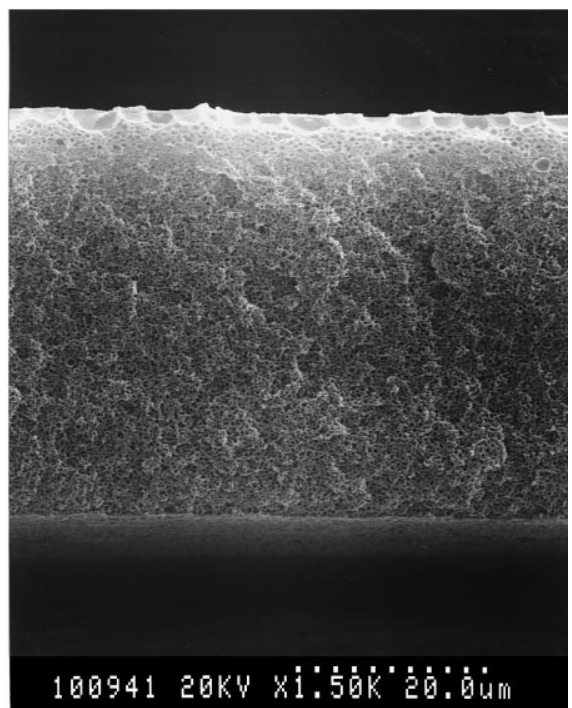

Fig. 4. SEM photographs of the EVAL membrane prepared with $1 \mathrm{~min}$ of absorption process at $21^{\circ} \mathrm{C}$ under the relative humidity of $75 \%$ followed by immersion into a water bath (a) top surface; (b) cross-section.

little water diffuses into the casting solution since it is a nonsolvent for EVAL copolymer. Consequently, a pronounced high polymer concentration resulting from rapid solvent outflow can be set up, which can be confirmed by a mass transport model described previously [11]. Finally, the interfacial composition of the membrane solution (polymer-rich phase on the binodal) and the interface of the coagulation bath (polymer-poor phase) are in equilibrium. Such a high polymer concentration brings the top layer into a very hard and stiff state so as to preclude the possibility of any porous morphology at the membrane surface.

After the skin layer is formed, nonsolvent continually diffuses through the toplayer to the sublayer. If the diffusion is sufficiently slow, solid-liquid demixing may take place first. In our previous study, the instantaneous demixing took place in this membrane formation process [11]. This suggests that the time for water molecules diffusing into the interior portion of the membrane solution is not long enough for solid-liquid demixing to start. Therefore, liquid-liquid demixing can precede solid-liquid demixing. Those liquid nuclei grow into micelles, which eventually phase-inverts into cellular pores or finger-like macrovoids.

In order to explain the effect of the absorption process on the EVAL membrane structure, a change in weight of the casting mixture under the relative humidity of $75 \%$ was determined. As is shown in Fig. 6, the weight of casting mixture increased rapidly with increasing the absorption time at room temperature. Since DMSO evaporates very slowly at room temperature and is very highly hygroscopic, this increase in weight is mainly caused by the absorption of water molecules into the casting mixture from the atmosphere at high humidity. Hence, polymer concentration did not increase to form a dense skin at the membrane surface. In contrast, the nucleus of polymer-poor phase occurred due to the rapid water absorption. When the absorption of water molecules further increased, the majority of water molecules diffused into the growing pores. Accordingly, the pores became large to form a beehive-like surface on the membrane. Kawai et al. found the absorbed water droplets contacted each other to form a water film on the membrane surface [16]. This suggests that most of water would be retained on the membrane surface and only little water molecules diffused into the sublayer. Hence, it created a slow formation rate for the sublayer in the nonsolvent absorption case. The slower the formation rate, longer was the evolution to liquid-liquid demixing of the sublayer. Consequently, the decrease of water moving into the sublayer allowed the solid-liquid demixing to proceed. 
(a)

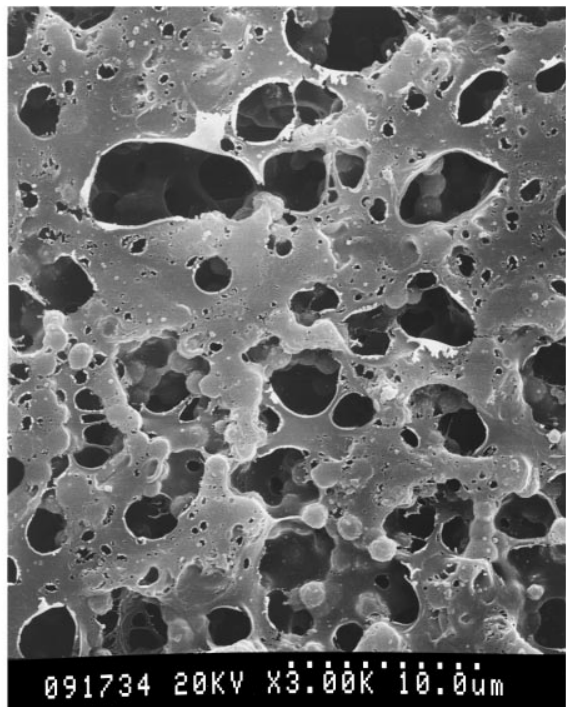

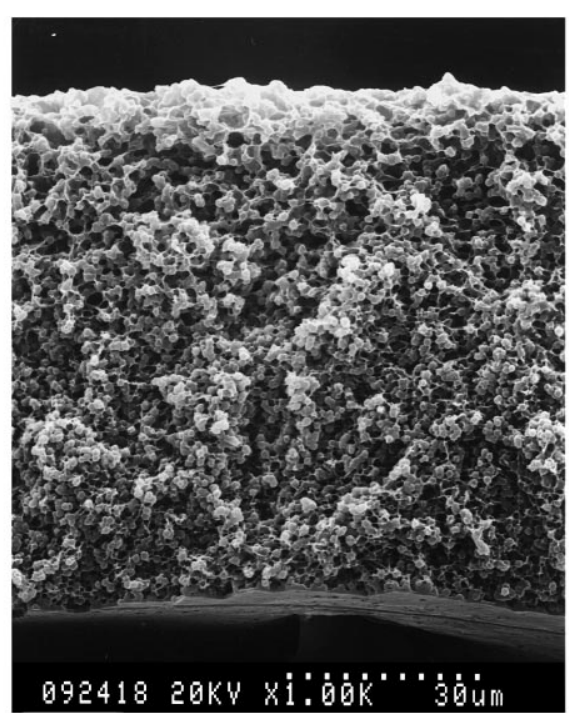

(c)

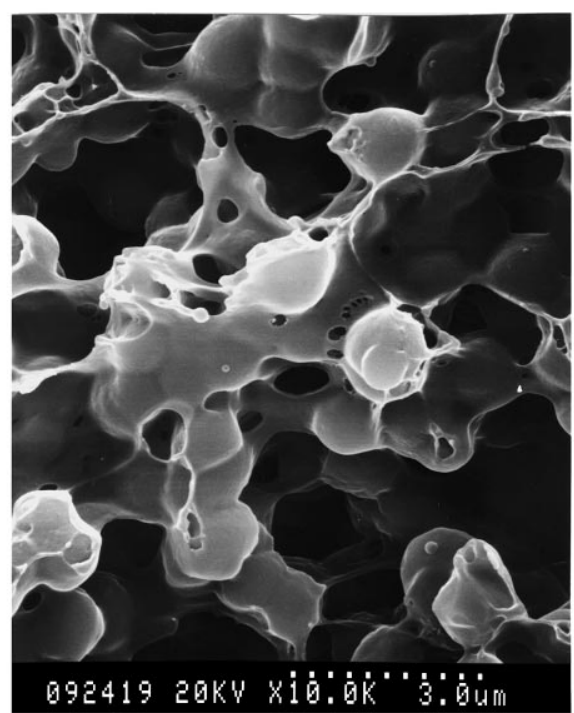

Fig. 5. SEM photographs of the EVAL membrane prepared with 3 min of absorption process at $21^{\circ} \mathrm{C}$ under the relative humidity of $75 \%$ followed by immersion into a water bath (a) top surface; (b) cross-section; (c) magnification of (b).

\subsection{The composition path for membrane with or without a nonsolvent absorption process}

The membranes prepared in this work indicate that a greatly different morphology can be associated with nonsolvent absorption during membrane formation. This difference clearly implies that they should have different composition paths in the phase diagram even though the membrane formation system with or without a nonsolvent absorption process has the same phase diagram. Especially, the possibility of crystallization can complicate the effect of nonsolvent absorption on the crystalline polymer membrane. Therefore, the rate of solvent-nonsolvent exchange is of the utmost importance for the membrane structure. Fig. 7 shows, qualitatively, the expected composition paths of toplayer and sublayer as membrane formation proceed with time. All the composition paths enter 


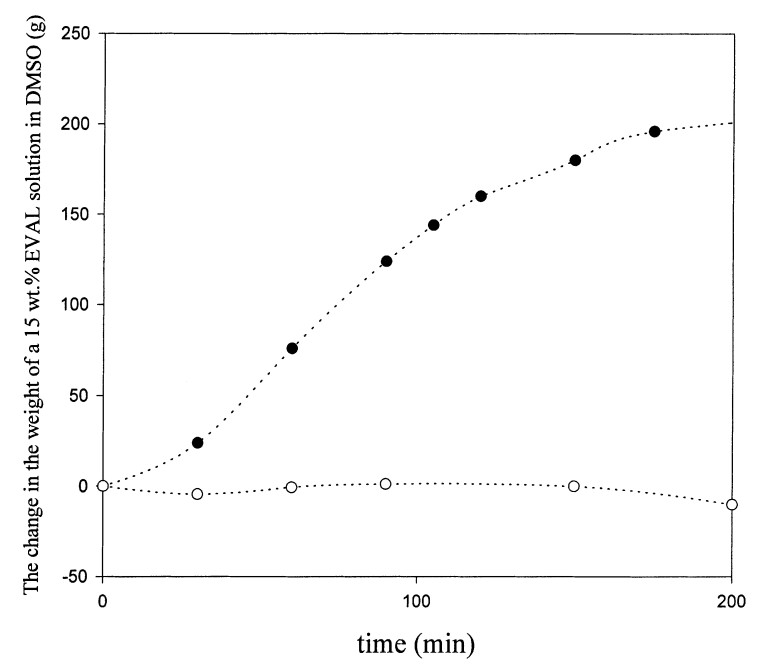

Fig. 6. The change in the weight of a $15 \mathrm{wt} \%$ ECAL solution in DMSO with time. ( $)$ under the relative humidity of $75 \%$ at $21^{\circ} \mathrm{C}, \mathrm{O}$ : under the relative humidity of $30 \%$ at $55^{\circ} \mathrm{C}$ ).

first the solid-liquid demixing region, irrespective of immersing EVAL solution into water or the absorption of water molecules into the casting mixture. Therefore, this difference in membranes prepared with or without nonsolvent absorption is discussed by taking into account the effect of the diffusion kinetics on the resulting membrane structure.

The composition path for membranes prepared by a direct immersion process follows the line ' $a$ ' in Fig. 7. The composition path of toplayer (a1) shows a steep slope to enter the binodal due to the sudden solvent outflow of the casting solution [11]. This results in a rapid increase in polymer concentration to favor a nonporous layer on the membrane surface. The sublayer structure is controlled by the diffusion rate of solvent and nonsolvent through the skin layer. Because the skin layer creates an additional barrier for solvent diffusion out of the membrane solution, the composition path of sublayer (a2) enters the binodal at a lower polymer concentration, resulting in a porous structure. Based on the above discussion and SEM observation (Fig. 2), their composition paths are different but are rapid enough, thus, crystallization does not occur until liquid-liquid phase separation has completely set in the membrane structure.

On the other hand, since DMSO evaporates very slowly at room temperature, the composition path for

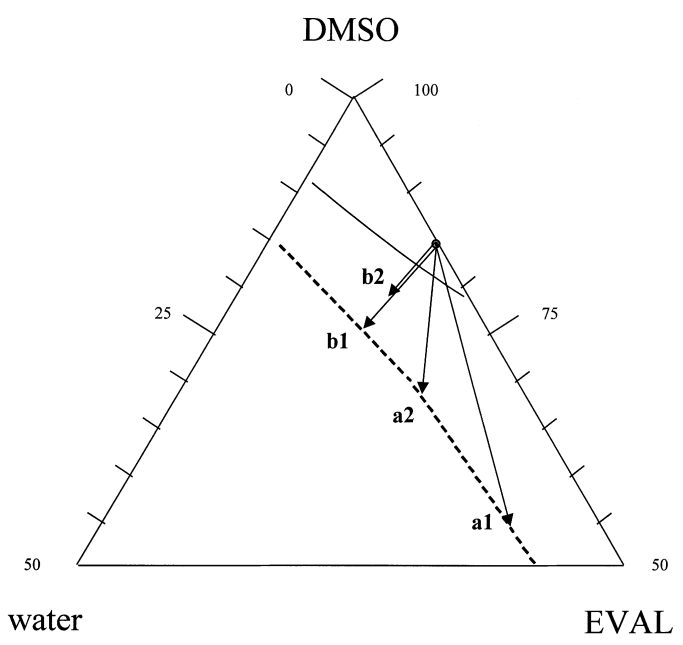

Fig. 7. Composition paths for preparation of membranes by. (a) a direct immersion into a water bath; (b) an absorption process (1: toplayer and 2: sublayer).

membranes prepared with the nonsolvent absorption process is expected to follow the line ' $b$ ' toward the water apex corresponding to Fig. 7. In addition, since DMSO is very highly hygroscopic, the condition for absorbing water is fast relative to the time necessary for solid-liquid demixing. Therefore, the composition path of toplayer (b1) enters the binodal to cause liquid-liquid phase separation and the result is beehive-like surface. Qualitative comparisons of the toplayer with the sublayer for membranes with the absorption of water molecules into the membrane solution indicate their formation kinetics are different though they have the same composition path due to water inflow only. Hence, the composition path of the sublayer (b2) can remain entirely in the solid-liquid demixing region and outside the liquid-liquid demixing region. A reasonable assumption is most of absorbed water molecules were kept on the membrane surface [16]. Therefore, the water molecules further moving into the sublayer are severely reduced. The reduction in the driving force for water inflow leads to a slow demixing rate in the sublayer to form a particulate morphology. Conversely, if the water absorption time is too short, the induction time for crystallization is not long enough. Consequently, after immersion of the casting solution into a water bath, liquid-liquid demixing may take place first or liquid-liquid demixing and crystallization occur simultaneously in the sublayer, 
(a)

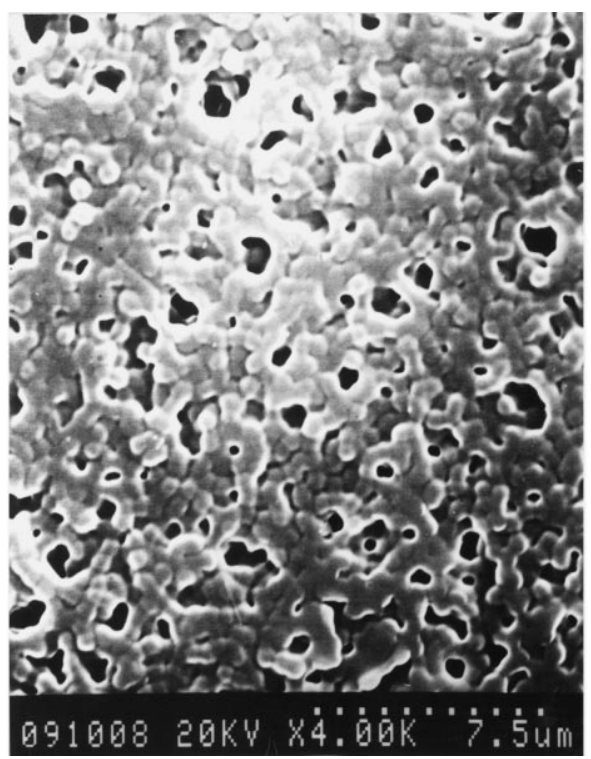

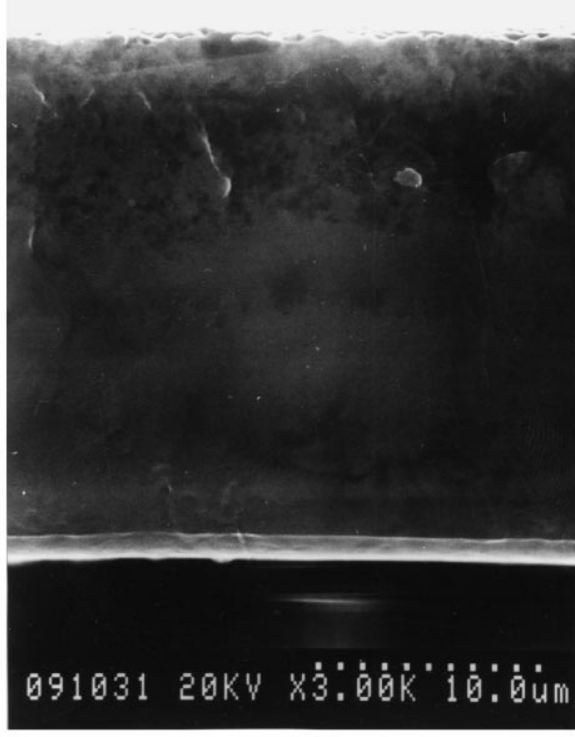

(b)

Fig. 8. SEM photographs of the EVAL membrane prepared with an absorption process under the relative humidity of $30 \%$ at $55^{\circ} \mathrm{C}$ followed by immersion into a water bath (a) top surface; (b) cross-section.

thus, two types of phase separation may occur during the membrane formation.

Moreover, the process of liquid-liquid demixing occurring on the membrane surface due to the rapid nonsolvent absorption will disappear at higher temperatures and at lower humidity. Fig. 6 shows the change in weight of a $15 \mathrm{wt} . \%$ of EVAL solution in DMSO with time at $55^{\circ} \mathrm{C}$ under the relative humidity of $30 \%$. The slight decrease in weight observed indicates that the amount of DMSO evaporated from polymer solution slightly exceeds the absorbed amount of water. Therefore, the composition path will change form the line $b$ to the line $a 2$ or $a 1$. In addition, the absorption rate became small due to lower humidity and higher temperature. It is obvious that the time available for the membrane within the solid-liquid demixing region is become longer for the lower water absorption rate and the longer distance in location of the phase boundaries. Hence, the crystallizationinduced particulate morphologies were found on the membrane surface; see Fig. 8. In fact, this membrane showed a particulate surface layer supported by a dense sublayer. It is suggested that only DMSO left the sublayer by evaporation instead of the exchange of solvent and nonsolvent. Hence, the composition path will follow the solvent-polymer axis, leading to a dense structure at the sublayer.

\section{Conclusions}

This work shows that both crystallization and liquid-liquid demixing occur on two different time scales in preparing EVAL membranes. Membranes prepared with a direct immersion into the coagulation bath had a dense skin layer with finger-like macrovoids in the sublayer. However, membranes prepared with the absorption of water molecules into the casting mixture from the atmosphere provided a porous top layer supported by a sublayer with particles in a narrow size distribution. This morphological difference arises from the fact that their courses and rates of precipitation are different. Considering the phase diagram and experimental results, membrane formation mechanism can be established. The toplayer and the sublayer of membrane with a direct immersion have different courses, but their rates are fast enough, thus, the thermodynamically possible crystallization does not occur until liquid-liquid phase separation has occurred. Hence, the structure con- 
trolled by liquid-liquid demixing is favored. On the contrary, the toplayer and the sublayer of membrane prepared by an absorption process have the same composition path, but their rates are different. The rapid nonsolvent inflow induces a porous toplayer. Conversely, the composition change in the sublayer is rather slow. Therefore, the liquid-liquid demixing does not occur in the sublayer.

\section{Acknowledgements}

The authors thank the National Science Council of the Republic of China for their financial support of project NSC 89-2216-E-002-006.

\section{References}

[1] M. Mulder, Basic Principles of Membrane Technology, Kluwer Academic press, Dordrecht, The Netherlands, 1991.

[2] J.G. Wijmans, H.J.J. Rutten, C.A. Smolders, Phase separation phenomena in solutions of poly (2,6-dimethyl-1,4phenyleneoxide) in mixtures of trichloroethylene, 1-octanol and methanol: relationship to membrane formation, J. Polym. Sci. Polym. Phys. 23 (1985) 1941-1955.

[3] A.M.W. Bulte, B. Folkers, M.H.V. Mulder, C.A. Smolders, Membranes of semicrystalline aliphatic polyamide nylon 4,6: formation by diffusion-induced phase separation, J. Appl. Polym. Sci. 50 (1993) 13-26.

[4] L.P. Cheng, A.W. Dwan, C.C. Gryte, Membrance formation by isothermal precipitation in polyamide-formic acid-water systems I. Description of membrane morphology, J. Polym. Sci. Polym. Phys. 33 (1995) 211-222.

[5] L.P. Cheng, A.W. Dwan, C.C. Gryte, Membrane formation by isothermal precipitation in polyamide-formic acid-water systems II. Precipitation dynamics, J. Polym. Sci. Polym. Phys. 33 (1995) 223-235.

[6] P. van de Witte, H. Esselbrugge, P.J. Dijkstra, J.W.A. van de Berg, J. Feijen, A morphological study of membranes obtained from the systems polylactide-dioxane-methanol, polylactide-dioxane-water, and polylactide- $N$-methyl pyrrolidone-water, J. Polym. Sci. Polym. Phys. 34 (1996) 25692578.

[7] L.P. Cheng, T.H. Young, L. Fang, J.J. Gau, Formation of particulate microporous poly(vinylidene fluoride) membranes by isothermal immersion precipitation from 1-octanol/dimethylformamide/poly(vinylidene fluoride) system, Polymer 40 (1999) 2395-2403.

[8] L.P. Cheng, H.Y. Lin, L.W. Chen, T.H. Young, Solute rejection of dextran by EVAL membranes with asymmetric and particulate morphologies, Polymer 39 (1998) 2135 2142.

[9] D.T. Lin, L.P. Cheng, Y.J. Kang, L.W. Chen, T.H. Young, Effects of precipitation conditions on the membrane morphology and permeation characteristics, J. Membr. Sci. 140 (1998) 185-194.

[10] T.H. Young, C.H. Yao, J.S. Sun, C.P. Lai, L.W. Chen, The effect of morphology variety of EVAL membranes on the behavior of myoblasts in vitro, Biomaterials 19 (1998) 717724.

[11] L.P. Cheng, T.H. Young, W.M. You, Formation of crystalline EVAL membranes by controlled mass transfer process in water-DMSO-EVAL copolymer systems, J. Membr. Sci. 145 (1998) 77-90.

[12] T.H. Young, L.P. Cheng, C.C. Hsieh, L.W. Chen, Phase behavior of EVAL polymer in water-2-propanol Cosolvent, Macromolecules 31 (1998) 1229-1235.

[13] T.H. Young, L.W. Chen, L.P. Cheng, Membranes with a microparticulate morphology, Polymer 37 (1996) 1305-1310.

[14] T.H. Young, L.Y. Chen, Y.H. Huang, W.Y. Chiu, L.W. Chen, Membranes with a particulate morphology prepared by a dry-wet process, Polymer 40 (1999) 5257-5264.

[15] H.C. Park, Y.P. Kim, H.Y. Kim, Y.S. Kang, Membrane formation by water vapor induced phase inversion, J. Membr. Sci. 156 (1999) 169-178.

[16] T. Kawai, Y.M. Lee, S. Yamada, Preparation of asymmetric porous membranes of poly (vinyl chloride), Polymer 38 (1996) 1631-1637.

[17] T. Uragami, Y. Ohsumi, M. Sugihara, Studies on syntheses and permeabilities of special polymer membrane: 38 Formation mechanism of finger-like cavities in membranes from cellulose nitrate and single solvent, Polymer 23 (1982) 999-1004.

[18] T.H. Young, J.Y. Lai, W.M. Yu, L.P. Cheng, Equilibrium phase behavior of the membrane forming water-DMSO-eval copolymer system, J. Membr. Sci. 128 (1997) 55-65.

[19] C. Cohen, G.B. Tanny, S.J. Prager, Diffusional-controlled formation of porous structures in ternary polymer systems, J. Polym. Sci. A-2 (17) (1979) 477-489.

[20] L. Yilmaz, A.J. McHugh, Modeling of asymmetric membrane formation I. Critique of evaporation models and development of a diffusion equation formalism for the quench period, $\mathrm{J}$. Membr. Sci. 28 (1986) 287-310.

[21] A.J. Reuvers, J.W.A. van der Berg, C.A. Smolders, Formation of membranes by means of immersion precipitation. Part I. A model to describe mass transfer during immersion precipitation, J. Membr. Sci. 34 (1987) 45-65.

[22] P. Radovanovic, S.W. Thiel, S.-T. Hwang, Formation of asymmetric polysulfone membranes by immersion precipitation Part I. Modeling mass transport during gelation, J. Membr. Sci. 65 (1992) 213-229.

[23] T.H. Young, L.W. Chen, A two step mechanism of diffusion-controlled ethylene vinyl alcohol membrane formation, J. Membr. Sci. 57 (1991) 69-81. 